Gómez-Sánchez, J.M., Ponz-Tienda, J.L. and Romero-Cortés, J.P. (2019). "Lean and BIM Implementation in Colombia; Interactions and Lessons Learned" In: Proc. 27th Annual Conference of the International. Group for Lean Construction (IGLC), Pasquire C. and Hamzeh F.R. (ed.), Dublin, Ireland, pp. 1117-1128. DOI: https://doi.org/10.24928/2019/0150. Available at: 〈www.iglc.net>.

\title{
LEAN AND BIM IMPLEMENTATION IN COLOMBIA; INTERACTIONS AND LESSONS LEARNED
}

\author{
Juan Martín Gómez-Sánchez ${ }^{1}$, José Luis Ponz-Tienda², Juan Pablo Romero-Cortés ${ }^{3}$
}

\begin{abstract}
Construction projects are afflicted by cost and time overruns, primarily due to inadequate information sharing among the project participants that causes productivity reductions in the Architectural, Engineering, and Construction (AEC) industry. Therefore, by working on different areas of its value chain, it is possible to increase the construction process productivity. Building Information Modelling (BIM) emerged as a digital platform through which project teams can share and manage project information efficiently, and Lean construction practices address the issue of improving coordination within a project team, smoothing workflow in construction projects through features that reduce waste and increase value.

The present research is an applied study that comprises the analysis of the integration of Lean practices and BIM through a real-life implementation in a residential project. This paper contributes to knowledge on BIM and Lean adoption by showing how lean practices reduce coordination-related issues within the project construction, and BIM adoption makes the benefits of Lean principles more visible. Additionally, the literature still lacks specific examples of simultaneous implementation of BIM and Lean for the first time in medium-sized construction companies. The purpose is to report the experiences, opportunities for improvement, and lessons learned from the implementation. Findings show that independent implementation only solves a part of the problem. However, simultaneous implementation exposed the team to more exceptional experiences and opportunities for improvement.
\end{abstract}

\section{KEYWORDS}

Lean construction and BIM Interaction, case study

MS Student, Civil and Envir. Engineering Dept., Univ. de los Andes, Bogotá, Colombia, (57-1) 3324312, jm.gomez14@uniandes.edu.co

$2 \mathrm{PhD}$, Assistant Professor, Civil and Envir. Engineering. Dept., Director of the Engineering and Construction Management Research Group., Univ. de los Andes, Bogotá, Colombia, (57-1) 3324312, jl.ponz@uniandes.edu

3 Project manager, Dep. of Civil and Environmental Engineering. Universidad de Los Andes, Bogotá, Colombia, (57-1) 3324312, jp.romero985@uniandes.edu.co 


\section{INTRODUCTION}

At the global level, a pattern of low productivity in the construction sector has become evident. The period between 1995 and 2015, evidences that the compound rate of growth of value added per worker in the sector was just $1.0 \%$, while the economy was $2.7 \%$, the gap is even more significant if compared to the industry manufacturing, where growth reached a rate of around 3.6\% per year in this same period (McKinsey, 2017). It fits highlight that at the regional level and even within of the companies that make up the sector finds quite a heterogeneity. In fact, Colombia is not an exception. In Colombia, disruptive production delivery methods and technologies can increase construction productivity between $50 \%$ and $60 \%$. For example, BIM has been reported to be in its experimentation stage for most AEC companies, but its adoption is continuously growing (Gómez-Sánchez et al., 2016). However, it is expected to grow even faster in the coming years due to the launching of the institutional platform for the articulation of actors around the digitization of the construction sector, BIM Forum Colombia, which seeks to standardize and disseminate BIM adoption nationally.

Additionally, according to Mossman (2018), Lean Construction would be more helpful to define Lean concerning its purpose than in its meaning. He concluded that Lean is "a practical collection of theories, principles, axioms, techniques, and ways of thinking that together and severally can help individuals and teams improve the processes and systems within which they work." Many different Lean principles are beneficial to the overall construction project's life cycle. On the other hand, BIM has been massively recognized as a platform which is related to improve the design-construction process through digitalization and has been defined as "not just a technology change, but also a process change" (Eastman et al., 2012).

Although these approaches are different initiatives, there exists a synergy between Lean and BIM that brings better results when implemented together than independently (Sacks et al., 2010). While BIM tackles the trustworthiness of information, Lean tackles the reliability of processes (Fosse et al., 2017). However, concerning the use of multidisciplinary performance models (BIM) of design-construction projects that includes work processes and organization of the design-construction team, a new concept was pioneered by the CIFE (Khanzode et al., 2006) at Stanford University: Virtual Design and Construction (VDC). VDC focuses on aligning new technology related to BIM with Lean Thinking and practices (Kunz \& Fischer, 2012) and VDC tools can be applied very effectively to accomplish the objectives of the Lean Production Delivery System (LPDS).

In this research, the authors proposed that the pursued value added could be achieved not only through the digitalization of the company's pilot's project traditional workflow because the current full potential for improvement of construction projects "can be achieved when the adoption of Lean and BIM adoption is integrated" (Sacks et al., 2010). Therefore, this paper discusses the lessons learned from the simultaneous implementation of Lean and BIM approaches in a case study in Colombia. The pilot project's owner wanted to generate the maximum possible amount of value added to their customers: provide a higher quality living unit, better customer service, reduce post-sales issues and brand 
recognition as an innovative company that highlights from other traditional construction companies. However, the methods to be applied to achieve the objective were not clear.

\section{RESEARCH BACKGROUND}

In terms of the scope of the implementation, although (Khanzode et al., 2006) and (Sacks et al., 2010) found and identified a high number of interactions between BIM and Lean, the pilot project's objectives were carefully set in order to experience parallel benefits and to avoid overwhelming the staff with many new concepts, working processes and tools. Additionally, the authors also considered the probability of underutilizing the tools and the interoperability limitations for the definition of the scope of the BIM implementation. BIM and Lean execution were made from the general manager perspective, and the objective of the implementation was to learn, plan and use both approaches in the entire project; however, this paper concentrates in the experiences of the excavation, foundation and structural construction phases.

Finally, by reviewing literature, examples of client's integration of Lean and BIM in real projects have been documented, measured and compared through different Lean and BIM maturity matrixes (e.g., Mahalingam et al., 2015; Fosse et al., 2017; Hamdi \& Leite, 2012; Bolpagni et al., 2017; Khan \& Tzortzopoulos, 2014). However, the literature still lacks specific examples of client's simultaneous implementation of BIM and Lean for the first time in a medium-sized construction company.

\section{METHODOLOGY}

The present research is an applied study that comprises the analysis of the integration of Lean practices and BIM tools through a real-life implementation in a construction company. The authors analyzed the 18 BIM functionalities interacting with the 24 Lean construction principles under the Interaction Matrix developed by Sacks et al. (2010), which proposes a conceptual framework for analyzing the interaction of the two transformative methodologies, Lean Construction, and BIM. In the current paper, the authors focused on a limited number of interactions applying them in a case study based on the exploration of a real-life project that is used to depict the relationships between BIM and Lean to report experiences specifically, opportunities for improvement and lessons learned from the implementation.

\section{CASE STUDY}

A medium-sized company, whose principal activities are fostering, marketing and constructing civil and real estate projects, had been planning to incorporate BIM and Lean management to their company. They decided to start the long journey with a pilot project in a residential building that consists of 25 stories, 3 basements, and 281 living units with a gross built-up area of $20.900 \mathrm{~m} 2$ in a lot of $1.500 \mathrm{~m} 2$ approximately. For accomplish this goal, the company hired as a consultant the Research Group of Engineering and Construction Management (INGECO) of Universidad de Los Andes, whose main goal was

to kick-off the Lean and BIM implementation using the pilot project, so the company could 
learn and acquire the capabilities and skills to continue it. For this, the team worked in three levels of hierarchy: a.) owners, b.) project managers and c.) designers/contractors.

From the design point of view, the project has 23 different apartment types. Although each of these units is standard, the significant variability of the project imposes great challenges for its production planning, production control, and logistics. The building also contemplates commercial uses in its ground floors. The procurement method was Design and Build (D-B) for the electric and hydraulic systems, and Design Bid and Build (D-B-B) for the other disciplines.

Although the company knew something about BIM tools, it had no experience with the application of VDC or Lean concepts in practice. The company is aiming to gain a benefit from being early adopters of Lean and BIM to directly reduce construction costs and obtain value added from their activity. Actually, according to theory, early adopters can be hugely benefited because a company can develop and maintain a significant competitive edge until the rest of the industry catches up (Sacks, Korb, \& Barak, 2018).

A description of the BIM planning, execution, and information management process are outlined below, as well as the Lean Culture dissemination in the project team and selected Lean tools that the team implemented. Nevertheless, the next section presents an analysis of the simultaneous implementation experiences of both Lean and BIM.

\section{BIM Planning}

According to Ramírez-Sáenz et al. (2018), the development of a BIM Execution Plan (BEP) is crucial for better planning and understanding in the management of BIM in a project. It should be the previous activity to the project's BIM modeling. Consequently, the team collaboratively created the BEP. For the pilot project, it had no contractual links with the designers or contractors yet. In the project's modeling process there were no more than three stakeholders involved directly. The team identified that the expected results of the VDC implementation were: to minimize field conflicts, to increase in productivity in the field, to anticipate material orders to keep workflow, to speed up verification of design and installed quantities and to follow up the construction process.

Additionally, breaking down project information has been a successful way of managing and controlling construction projects efficiently in the past decades. A variation of the Work Breakdown Structure (WBS) concept, a Model Breakdown Structure (MBS) was manually ${ }^{4}$ carried in this project although an automated method proposal exists in recent literature (Rischmoller et al., 2017). The MBS was defined in the BEP as well. The main objectives of the MBS were to:

- Structure information to aid comprehension trying to adapt the BIM model to the project's WBS to facilitate Quantity Take-off (QTO)

- Assign work responsibilities related to the BIM activity

- Create flexible models to be easily navigated by on-site personnel

- Facilitate automated design coordination by allowing to have small and manageable groups of parametric objects

\footnotetext{
${ }^{4}$ Due to limitations in the software
} 
- Maintain virtual files of a manageable size to use them on ordinary laptop computers located on site

\section{BIM Execution}

As stated above, once the implementation objectives and scope were collaboratively defined, the implementation was carefully planned to ensure a paced adoption that matched the employee's gradual change in their mindset and their technical capabilities. Therefore, the first step toward implementing VDC was to introduce BIM as a coordination tool where conceptual and detailed designs were 2D-CAD based. The coordination was automated using Autodesk Navisworks and weekly construction detailing, and coordination meetings were conducted at the site office to discuss important design clashes with the aim of correcting the construction plans, being Navisworks the pivot point in each meeting. Nevertheless, coordination BIM models were provided to builders, but they were not required to use them. Because of this reason, there was an inevitable interruption in the flow of information (as well as in the traditional workflow) that could be addressed in the next step of the implementation (in a future project), by adopting BIM in design and construction and by having a Common Data Environment (CDE). The company should seek to ensure a unique database of information and that all information delivered to the construction site comes directly from the coordinated model.

The Architectural, Structural, Mechanical, Electrical, Plumbing (MEP) and Furniture design drawings were prepared using 2D CAD. The MEP contractors did not have the maturity to properly model their systems themselves nor their design were hired to be BIMbased. Therefore, the company's architect and the Research Group's ${ }^{5}$ engineers developed the 3D model of all of them with a maturity level that ranged from LOD 300 to 350 (BIMForum, 2018) based on 2D CAD design drawings and an A360 federated model was then used to build and share a Virtual Reality (VR) model.

Apart from the automated clash checking, the project obtained great benefits from virtual walkthroughs. They were carried out in the federated model with rendered textures and were a valuable help in the process of design checking and construction planning. Referring to design checking, they drastically facilitated the understanding of the interaction of the different systems. Additionally, walkthroughs allowed the team to detect errors that were not geometric clashes but helped to rethink design specifications regarding its functionality during operation, aesthetics, ease of installation, etc. By contrast, for construction planning, they enabled the team to plan logistics, prevent irrelevant crew motions, placement of equipment and materials, and evaluate labor at the site to improve safety.

\section{BIM Information Management}

Once the parametric building's model culminated, the idea was to start building the model. We refer exactly to raising the dimension of the 3D model. Information management refers to the Bill of Quantities (BOQ) preparation through the extraction of coordinated material quantities (5D) and building schedules simulations to evaluate constructability (4D). However, the project's team tried to begin with the end in mind, and the BEP had each use

\footnotetext{
${ }^{5}$ Research Group of Engineering and Construction Management (INGECO) of Universidad de Los Andes
} 
that the BIM models will have; otherwise, raising the dimension without having planned future BIM uses would involve much rework.

As for the 5D, material quantities were extracted directly from the coordinated Revit models and exported to an excel file to prepare Bill of Quantities (BOQ). Subsequently, each quantity extracted from the model was compared with those calculated from $2 \mathrm{D}$ drawings and analyzed, until we obtained a reliable result.

Finally, for 4D simulations, we followed a procedure that was hindered by interoperability because we created the schedule in Plexos but later converted to excel in order to import it on Navisworks Manage, which was synchronized with previously imported models to perform real-time simulations for construction sequencing. The most profitable part of this process was to evaluate different excavation alternatives, validate the construction process of the structure, and standardize the production in the typical floors.
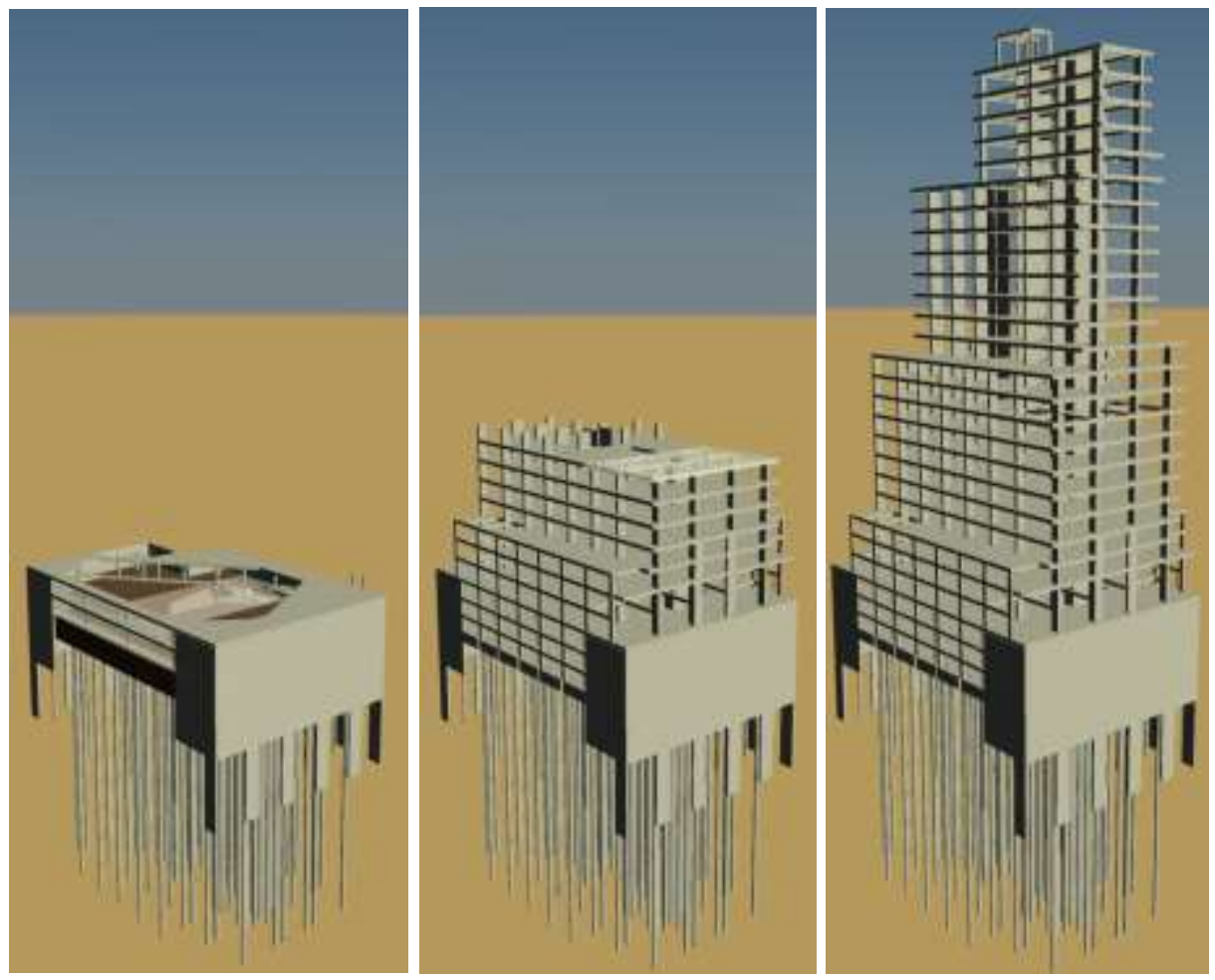

Figure 2: Autodesk Navisworks' 4D simulation

\section{Lean Culture}

While the parametric modeling of the building was in process, a Lean training strategy pretended to build a Lean culture in the organization and the project's participant's mindset. First, Sacks et al. (2010) and Hackler et al. (2017) concluded from their case study that although training on Lean tools is necessary, it is more important to create a culture based on the understanding of the Lean vocabulary, principles, and goals. Therefore, company's owners, management team and contractors started to learn the fundamentals ideas of Lean and then, the company focused on making the training interactive and on encouraging 
people to enhance collaboration, to express their concerns and to make reflections on how the learning experiences connected with the daily situations in the field.

\section{Lean Tools}

The main Lean tools that have been used in the project are the Last Planner System (LPS), Location Based Management (LBM), root cause analysis, 5S, Plus-Delta, and some other tools to promote visual management and establish the Lean principles in the project's team.

The scheduling process was carried out using an integration of Location-Based Management (LBM) and Last Planner System (LPS) because we were looking to benefit from this integration due to the combination of the social process of LPS with the technical tools of LBMS (Seppänen et al., 2015). Master schedule belongs to the domain of LBMS, and it was carried out using Plexos Project ("Plexos Project," 2019), a collaborative and flow-centered software (figure 1) capable to schedule production using Lines of Balance. Furthermore, a weekly planning and production control are clearly within the domain of LPS. The LPS and Lines of Balance work well together. From the beginning, a general Location Breakdown Structure was collaboratively defined. Additionally, LBM set additional modeling requirements to the BIM modeling team so that it allowed further BIM-based project and production control.

The Lookahead and Weekly Work Programs (WWP) were directly supported by the BIM models, and Senda Matrix Excel's extension was used (Gutierrez-Bucheli et al., 2017). Lean implementation was always framed by the concept that construction is a social activity, and the indicator that measured the fulfillment of commitments was established and became common understanding within the team was the PPC (Percent of Promises Completed). Once the implementation process of the WWP meetings for production control had started, autonomously, the project management team concluded that the PPC as key performance indicator was right, but it could be part of a more holistic project's performance indicator. The team realized that although the PPC considered the social process of collaborative planning, there was the possibility of weighting with each contractor's payment compliance to their employees, compliance to industrial safety requirements and cleanliness on the job site. In other words, we understood that the company was starting to adopt continuous improvement as part of the organizational culture.

\section{LEAN AND BIM; INTERACTIONS AND LESSONS LEARNED}

According to the Lean-BIM interaction matrix (Sacks et al., 2010), the project team experienced ten synergies between Lean-BIM and concluded that there are a few more with a high potential of application that the team can pursue with a little more maturity of the project team and additional minimum investment.

Restriction assessment: regarding the different experiences, the modeling process revealed additional constraints to the project planners due to the lack of rigor that existed in the detailed design drawings. As a result, the project team was able to discuss constraints well in advance of Lookahead or WWP meetings and day-to-day execution. "Early bad news are always good news" (Mossman, 2015). Also, the ease of creation of different detailed additional views of the project allowed more information to be available to make 
decisions and evaluate more constraints. So far, reprocessing has been avoided, and it is expected almost to eliminate rework in the future. In other words, it is related to the interaction two of the matrix.

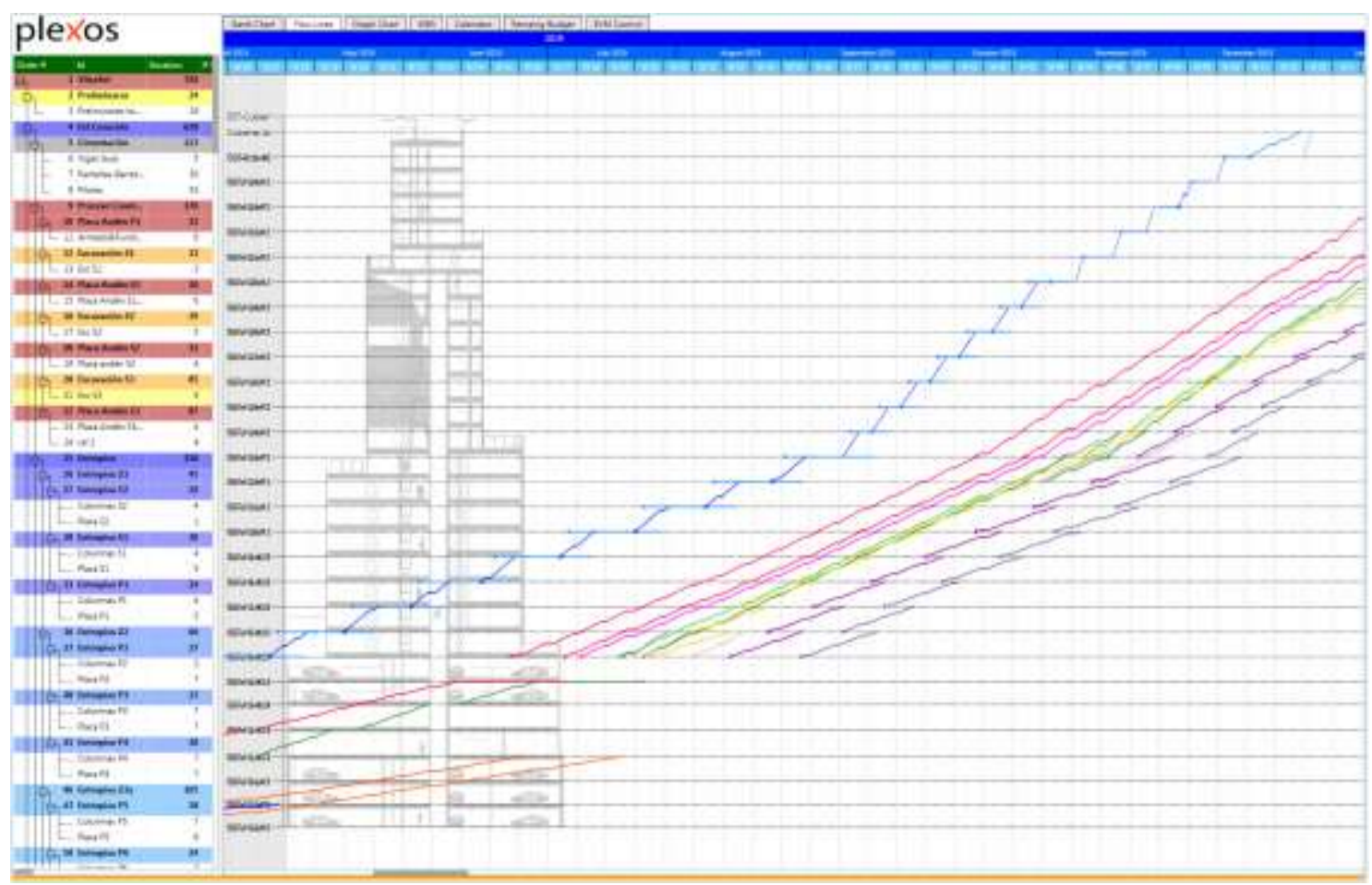

Figure 1: Project's Lines of Balance in Plexos Project (2019)

Team empowering: although designers did not work based on BIM, parametric models simplified design comprehension process to all contractors in a way that made planning more realistic, especially on behalf of project management (interactions 3 and 4 of the matrix). For example, the basement's water storage tank design presented some constructability issues that were discovered using the model and allowed the team to decide by consensus about the solution. Besides, interactions among the excavation process, concrete slabs, MEP systems, and the foundations were crucial to have a smooth flow of production. Additionally, although BIM was implemented in the project when the architectural design was utterly defined, the model's different options of visualization and management empowered the other stakeholders to propose minor or medium modifications that would improve their client's requirements (the next in line in the production process).

Virtual Gemba walks: virtual walkthroughs of integrated models (A360) helped stakeholders connect dots in design revisions. Because it does not require advanced BIM technical knowledge and does not require any software licenses (Navisworks Freedom), it can be a high-priced tool in low mature implementation projects. Apart from doing it in real life, it is beneficial to be able to virtually walk around the building in the different phases of the construction to potentiate the decision making of design and execution. The implementation has always sought to include visual management as a principle, 
permanently looking to positively impact system one of the human brain, which "operates quickly and automatically, without much effort, to make simple decisions" (Koskela et al., 2018). Interactions 6 and 12 of the matrix depicts them.

Design alternatives and construction simulation: as part of the evaluation of different design-execution options, the team developed a simulation of the excavation process alternatives because it had a restrictive constructive process due to particular soil characteristics. Similarly, we also created animations of the production sequences of foundations, structure, façade and interior walls in the same way (4D modeling) to evaluate constructability and guide the contractors on how to carry out activities in each project's zone and area. Interactions 7, 17, 40 of the matrix highlights these synergies.

Visual and automatic clash detection: the process of clash detection (interactions 12 and 24) performed in this project was considerably quick since test creation and execution followed a clear clash detection protocol, defined in the initial stages of the BEP. However, the design correction process was very time-consuming and took much effort. The result was practical, but it was not efficient at all because the contractors did not manipulate the models directly. The experience from this project shows that each designer should make the corrections of their disciplines so that there is a smooth flow of coordination in the preconstruction phase and the expert of each discipline conceives each solution alternative. Additionally, in some cases where time or BIM-trained personnel was available, contractors made 2D-based coordination due to fear and lack of full knowledge of BIM tools.

Automatic quantity take-off: undoubtedly, the automation of quantity extraction based on a BIM model (interaction 10) is one of the most significant benefits found in this synergy since it seeks to save time spent in unproductive tasks while reducing the subjectivity associated with this process (reduce variability). Not only was it used to validate budgeted quantities in the preconstruction phase, but also it was possible to calculate approximately $70 \%$ of the quantities executed on site. Additionally, after clash detection meetings, designs were adjusted. Estimated quantities kept updated because they maintained the original link to the quantity files. It is fundamental that the constructive process has been defined from the beginning of the project so that the model reflects the reality of execution and progress control quantification does not require additional effort. One experience from this project is that in Colombian industry there is an evident lack of standardization of quantity estimation criteria, which is why BIM tools are still inefficient due to this variability. However, BIM estimation tool's customization is possible with APIs, but they require advanced technical BIM capabilities.

BIM base scheduling and controlling: Last Planner System's meetings relied visually on BIM models. Besides, each weekly report contains a graphic preview of the project's current status, where visual information flows through all the project stakeholders, as well as the current restrictions and commitments. LPS and BIM require a minimum level of team collaboration. No matter the level of BIM implementation, it is a collaborative process. The more mature is the process, the more collaboration between professionals is required, and BIM allowed each planning meeting to be more comprehensible for the entire team. In this project, BIM modeling started one month before construction. Therefore, the lookahead plan subordinated the detailed modeling process (increasing the LOD). Keeping 
detailed modeling ahead of execution according to the needs of medium-term planning had a positive result in this project.

\section{CONCLUSIONS}

Each (BIM and Lean) implementation only solves a part of the problem. However, simultaneous implementation exposed the team to more exceptional experiences, frustrations, opportunities for improvement and lessons learned.

First, Lean requires sharp facilitator leadership and other "soft" skills that will allow motivation and encouragement to change the team's mindset towards new ways of working. Apart from that, the reverse phase scheduling (pull system) that has come to be closely associated with lean production is not always the best alternative, especially in lean construction pilot projects. On the contrary, the selection of the best method should depend on each stage of production, so the applied methods are mixed push-pull systems, as it happened in the present case study.

Second, if BIM tools are not properly implemented and managed, they can make a process more difficult and unstable. The availability of technical expertise will encourage BIM adoption in construction companies. Additionally, many BIM functionalities improved the flow of planning construction processes. In fact, as described by Sacks et al. (2018), the project underwent that BIM makes construction leaner even when lean was not the explicit intent in a certain activity. Additionally, from the client perspective, the BIM based design requires more caution and meticulousness from the designer, obtaining a better result in the project's earlier phases. For this reason, the authors consider that is justified the design prices increment due to the use of this methodology.

Third, the contracts and the BEP were aimed to gain the best results for the pilot project; however, to have these results, it is was important to consider the designs/contractors' skills and capabilities and not just the owners' and project managers'. Taking into account that the designers/contractors group were external to the company and had a low maturity level, assuring their participation in the training process would have had the expected outcome. As well, the learning experiences regarding Lean and BIM could have been translated into other projects.

Finally, regarding lessons for construction companies starting the implementation, companies should develop a maturity assessment before the implementation in order to benchmark their present capabilities and allow them to plan for the future, and achievements can be measured and controlled. Although it is better to implement simultaneously, the level of training, frustrations, and risks of failure are much higher. Moreover, the project team must prepare to cope in the best possible way these possibilities.

\section{ACKNOWLEDGMENTS}

The authors would like to thank the company's management team for their valuable teachings and time as well as to the support provided by the Research Group of Engineering and Construction Management (INGECO) at Universidad de los Andes. 


\section{REFERENCES}

BIMForum. (2018). The Level of Development (LOD) Specification 2018.

Bolpagni, M., Burdi, L., \& Ciribini, A. L. C. (2017). Integration of Lean Construction and Building Information Modeling in a Large Client Organization in Massachusetts. In K. Walsh, R. Sacks, \& I. Brilakis (Eds.), Proceedings for the 25th Annual Conference of the International Group for Lean Construction (pp. 79-86). Heraklion, Greece. https://doi.org/10.24928/2017/0311

Eastman, C. M., Teicholz, P., Sacks, R., \& Liston, K. (2012). BIM Handbook: A Guide to Building Information Modeling for Owners, Managers, Designers, Engineers and Contractors V2.0 (2nd ed., Vol. 53). John Wiley \& Sons. https://doi.org/10.1017/CBO9781107415324.004

Fosse, R., Ballard, G., \& Fischer, M. (2017). Virtual Design and Construction: Aligning BIM and Lean in Practice. In Proceedings for the 25th Annual Conference of the International Group for Lean Construction (pp. 499-506). Heraklion, Greece. https://doi.org/10.24928/2017/0159

Gómez-Sánchez, J. M., Rojas-Quintero, J. S., \& Aibinu, A. A. (2016). The Status of BIM Adoption and Implementation Experiences of Construction Companies in Colombia. In M. C. Mayorga \& J. L. Ponz-Tienda (Eds.), VII Elagec (pp. 601-611). Bogotá: ELAGEC 2016 Comité Organizador.

Gutierrez-Bucheli, L. A., Romero-Cortés, J. P., Mayorga-Calderón, M. C., LondoñoAcevedo, M. C., \& Ponz-Tienda, J. L. (2017). Senda Matrix software as a control and planning tool for project delivery in civil engineering. In Proceedings of INTED2017 Conference (pp. 3315-3322). Valencia, Spain. https://doi.org/10.21125/inted.2017.0843

Hackler, C., Byse, E., Reed, D., \& Alves, T. C. L. (2017). Building a Lean Culture. In Proceedings for the 25th Annual Conference of the International Group for Lean Construction (pp. 309-316). Heraklion, Greece. https://doi.org/10.24928/2017/0301

Hamdi, O., \& Leite, F. (2012). BIM and Lean Interactions From the BIM Capability Maturity Model Perspective: a Case Study. In Proceedings for the 20th Annual Conference of the International Group for Lean Construction.

Khan, S., \& Tzortzopoulos, P. (2014). Effects of the interactions between LPS and BIM on workflow in two building design projects. In Proceedings for the 22nd Annual Conference of the International Group for Lean Construction (Vol. 1, pp. 933-944). Oslo, Norway. https://doi.org/10.1016/j.autcon.2010.09.013

Khanzode, A., Fischer, M., Reed, D., \& Ballard, G. (2006). A Guide to applying the principles of virtual design \& construction (VDC) to the lean project delivery process. CIFE Working Paper \#093. Stanford, CA. https://doi.org/10.1016/S02105691(08)70898-2

Koskela, L., Tezel, A., \& Tzortzopoulos, P. (2018). Why Visual Management? Proc. 26th Annu. Conf. Int. Gr. Lean Constr., 250-260.

Kunz, J., \& Fischer, M. (2012). Virtual Design and Construction: Themes, Case Studies 
and Implementation Suggestions. Stanford, CA.

Mahalingam, A., Yadav, A. K., \& Varaprasad, J. (2015). Investigating the Role of Lean Practices in Enabling BIM Adoption: Evidence from Two Indian Cases. J. Constr. Eng. Manag., 141(March), 1-12. https://doi.org/10.1061/(ASCE)C0.1943-7862

McKinsey. (2017). Reinventing Construction: A Route To Higher Productivity. McKinsey \& Company.

Mossman, A. (2015). Last Planner - collaborative short-term production planning.

Mossman, A. (2018). What is lean construction : another look - 2018. Proc. 26th Annu. Conf. Int. Gr. Lean Constr., 1240-1250. https://doi.org/10.24928/2018/0309

Plexos Project. (2019). Retrieved from https://www.plexosproject.com/index.php

Ramírez-Sáenz, J. A., Gómez-Sánchez, J. M., Ponz-Tienda, J. L., Romero-Cortés, J. P., \& Gutierrez-Bucheli, L. A. (2018). Requirements for a BIM execution plan ( BEP ): a proposal for application in Colombia. Build. Manag., 2(2), 05-14. https://doi.org/10.20868/bma.2018.2.3763

Rischmoller, L., Dong, N., Fischer, M., \& Khanzode, A. (2017). BIM Breakdown Structure. In Proceedings for the 25th Annual Conference of the International Group for Lean Construction (pp. 389-396). https://doi.org/10.1016/j.jth.2013.12.003

Sacks, R., Korb, S., \& Barak, R. (2018). Building Lean, Building BIM: Improving Construction the Tidhar Way. Routledge.

Sacks, R., Koskela, L., Dave, B. A., \& Owen, R. (2010). Interaction of Lean and Building Information Modeling in Construction. J. Constr. Eng. Manag., 136(9), 968-980. https://doi.org/10.1061/(ASCE)CO.1943-7862.0000203

Seppänen, O., Modrich, R.-U., \& Ballard, G. (2015). Integration of Last Planner System and Location-Based Management System. In Proceedings for the 23rd Annual Conference of the International Group for Lean Construction (pp. 123-132). Perth, Australia. 